\title{
A dietary flavone confers communicable protection against colitis through NLRP6 signaling independently of inflammasome activation
}

\author{
K Radulovic ${ }^{1,2,6}$, S Normand ${ }^{1,6}$, A Rehman ${ }^{3,6}$, A Delanoye-Crespin ${ }^{1}$, J Chatagnon ${ }^{1}$, M Delacre ${ }^{1}$, \\ N Waldschmitt ${ }^{1}$, LF Poulin ${ }^{1}$, J Iovanna ${ }^{4}$, B Ryffel $^{5}$, P Rosenstiel $^{3}$ and M Chamaillard ${ }^{1}$
}

Flavones represent a class of polyphenols that are found in many plant-derived food sources. Herein, we provide evidence that the anti-inflammatory and antiproliferative effect of the flavone apigenin relies on the regulation of the gut microbiota by the NOD-like receptor family pyrin domain containing 6 (NIrp6). When challenged by dextran sulfate sodium (DSS) in drinking water, mice were protected against colitis upon cohousing with apigenin-treated animals. In contrast, the protective effect was lost in the absence of NIrp6. Sequencing of the 16S ribosomal RNA gene revealed a shift in the composition of the gut microbiota in apigenin-treated mice that was not observed in the absence of Nlrp6. Equally important, we find that the antiproliferative effect of apigenin was dominantly transmitted after cohousing, while being compromised in NIrp6-deficient mice. In contrast, the symptoms of colitis were alleviated upon apigenin administration even in the absence of either caspase-1/11 or Asc. Collectively, these data indicate that apigenin modulated an inflammasome-independent mechanism by which Nirp6 reprograms the gut microbiota for protecting mice against colitis. Our study highlights a modulation of the Nlrp6 signaling pathway by a prominent constituent of the human diet that may point toward improved ways to treat inflammatory bowel diseases.

\section{INTRODUCTION}

The gut is inhabited by the microbiota that consists of highly diverse bacterial microorganisms with widely differing living requirements. Notably, the genome of the trillions of bacteria residing within the lumen of the intestine encompasses several biosynthetic pathways that are primarily influenced by drug intake, hygiene, and nutrition. One can consider the microbiota as our 'extended self,' inasmuch as this multicellular organ consumes, stores, and redistributes energy harvested from nutrients since the earliest days of life onwards. ${ }^{1}$ Throughout the host's entire life, this paradigm is also well exemplified by the beneficial impact of some bacteria on postnatal maturation of the immune system. With this reductionist organ centered view in mind, symbiosis is defined as an association between organisms of two different species in which each is benefited.
Conversely, instability in the composition of gut microbial communities (commonly referred to as dysbiosis) has been linked to alterations in the metabolic activity of the gut microbiota in several common human illnesses with increasing socioeconomic impacts. In particular, inflammatory bowel disease is associated with a lower abundance of several Firmicutes, ${ }^{2,3}$ including some Lactobacilli that are capable of metabolizing tryptophan into aryl hydrocarbon receptor ligands. ${ }^{4}$ However, it remains elusive how to effectively restore the symbiotic host-microbiota interactions in patients suffering from such chronic inflammatory disorder.

Herbal-based supplements are used for many years as nutraceuticals for the treatment of various symptoms related to inflammatory bowel disease such as pain and diarrhea. Among nutraceuticals, dietary flavonoids are the largest class of

${ }^{1}$ Univ. Lille, CNRS, Inserm, CHRU Lille, Institut Pasteur de Lille, U1019-UMR 8204-CIIL-Centre d'Infection et d'Immunité de Lille, Lille, France. ²University Hospital Basel and University of Basel, Department of Biomedicine, Basel, Switzerland. ${ }^{3}$ Institute of Clinical Molecular Biology, Christian-Albrechts-University and University Hospital SchleswigHolstein, Campus Kiel, Kiel, Germany. ${ }^{4}$ Centre de Recherche en Cancérologie de Marseille, Inserm U1068, CNRS UMR 7258, Aix-Marseille Université and Institut PaoliCalmettes, Parc Scientifique et Technologique de Luminy, Marseille, France and ${ }^{5}$ CNRS, UMR7355; Experimental and Molecular Immunology and Neurogenetics, University of Orleans, France and IDM University of Cape Town, Orleans, France. Correspondence: M Chamaillard (mathias.chamaillard@inserm.fr)

${ }^{6}$ These authors shared first authorship. 
polyphenols that have been known as pigments for over a century (named from the latin word flavus meaning yellow). Besides their role in plant pigmentation and flavor, their common structure is a skeleton of diphenylpropane that confers a diversity of biochemical functions on plant physiology, including seed maturation, dormancy, and protection from oxidative stress, ultraviolet radiation, phytopathogens, and herbivores. Meanwhile, a plethora of epidemiological studies indicated that diets rich in some subclasses of dietary flavonoids are largely responsible for overall metabolic and cardiovascular health of humans. ${ }^{5-8}$ Notably, risk of developing colorectal cancer is lowered among individuals who regularly drink green tea, while being enhanced as a consequence of a lowered intake of flavonoids. ${ }^{9}$ Similarly, a lower risk of prostate cancer in men and of breast cancers in postmenopausal women was associated with intake of apigenin (4',5,7-trihydroxyflavone). Apigenin is a widely distributed secondary metabolite throughout the plant kingdom with a 2-phenylchromen-4-one (2-phenyl-1-benzopyran-4-one) backbone, representing an aglycone of several naturally occurring plant glycosides. The pharmacological properties of apigenin also include oxidative effects on human colorectal cancer cells that may result in senescence and wound healing. ${ }^{10}$ Furthermore, supplementation of the diet with the flavone apigenin contributes to the maintenance of gut barrier function in several preclinical models of either acute or chronic colitis. ${ }^{11-14}$ Nevertheless, it remains poorly understood how the apigenin exerts its antiinflammatory effect apart from its direct radical scavenging properties. Herein, we provide evidence that it could modulate the composition of the gut microbiota through the NOD-like receptor family pyrin domain containing 6 (Nlrp6) protein for protecting mice from acute colitis.

\section{RESULTS}

\section{Apigenin protects mice from acute colitis}

The physicochemical properties relevant to human health of the ingested apigenin are primarily determined by its metabolism and bioavailability. To overcome this issue, we assessed the potential effect of intraperitoneal administration of apigenin on the resolution of acute colitis. To this end, we made use of a preclinical model of intestinal inflammation that is induced by administration in the drinking water of dextran sodium sulfate (DSS) for 7 days in mice. This sulfated polysaccharide is known to promote epithelial tissue disruption that leads to microbial translocation and increased secretion of key signals of the acute phase response by infiltrating inflammatory cells. As expected, $\sim 15 \%$ decrease of the initial body weight was observed in wild-type mice at 9 days after DSS administration (Figure 1a). In contrast, apigenin administration improved body weight loss (Figure 1a) and effectively alleviated the shortening of colon length in response to DSS (Figure 1b). Foremost, the level of the proinflammatory cytokine interleukin-1 $\beta$ was lowered upon systemic administration of apigenin (Figure 1c), as what was observed when adult wild-type mice were fed with an
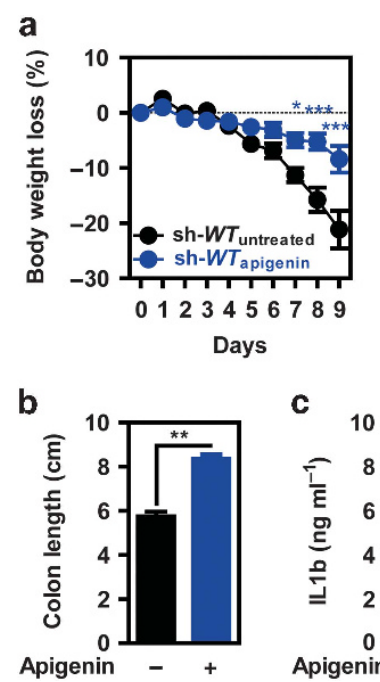

c

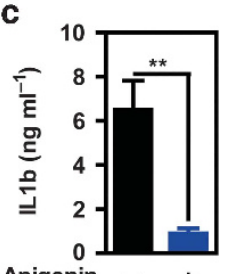

Apigenin - +
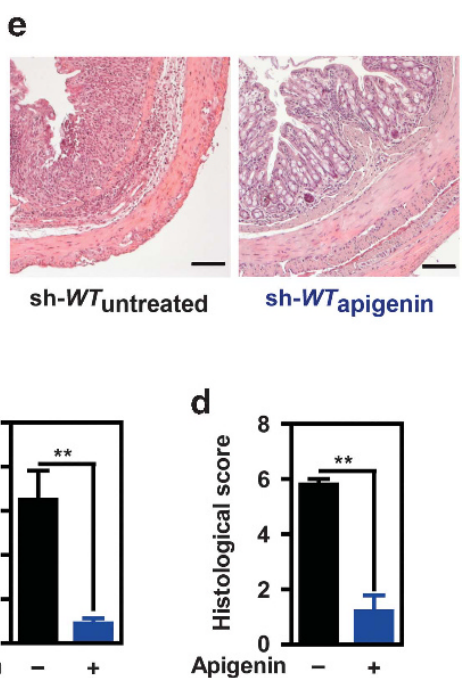

Figure 1 Systemic administration of apigenin protects mice from colitis. Single-housed wild-type mice ( $W T_{\text {apigenin }}$ in blue) were treated with apigenin at days 7 and 3 before induction of colitis by $2 \%$ dextran sulfate sodium (DSS; w/v). (a) The body weight loss was daily monitored in sh$W T_{\text {apigenin }}$ and compared with the one of five untreated wild-type C57BL6/J mice (sh- $W T_{\text {untreated }}$ in black). At day 10 , the (b) colon lengths, (c) interleukin-1 $\beta$ (ll1b) levels in colonic explants, (d) histological scores, and (e) representative hematoxylin and eosin (H\&E)-stained colonic sections are depicted. Error bars show s.e.m. Statistical significance was determined by using either two-way analysis of variance (ANOVA) with Bonferroni corrections for body weight loss or Mann-Whitney test as follows: ${ }^{\star} P \leq 0.05,{ }^{* \star} P \leq 0.01$ and ${ }^{* \star \star} P \leq 0.001$. Scale bar $=100 \mu \mathrm{m}$. Results are representative of three independent experiments.

apigenin-enriched diet. ${ }^{12}$ Consequently, histological examination of the hematoxylin and eosin-stained sections of the colon revealed a markedly reduced severity of colitis in apigenintreated mice (sh- $\left.W T_{\text {apigenin }}\right)$ when compared with controls (Figure 1d), as illustrated by erosion of the colonic crypts and massive infiltration of inflammatory cells (Figure 1e). Collectively, preventive administration of apigenin was found to improve acute signs of colitis in the wild-type setting as what was observed with other flavones. ${ }^{15}$

\section{Co-housing experiments revealed that the anti- inflammatory effect of apigenin is communicable to adult wild-type mice}

To assess whether the gut microbiota may contribute to the protective effect of apigenin against colitis, nontreated and apigenin-treated mice were cohoused for 3 weeks before DSS challenge (ch- $W T_{\text {untreated }}$ and ch- $W T_{\text {apigenin }}$ respectively). Unexpectedly, the body weight loss of either ch- $W T_{\text {untreated }}$ or ch$W T_{\text {apigenin }}$ mice was reduced to a similar extent (Figure 2a). Consequently, shortening of the colon was improved among cohoused mice (Figure 2b) as what was observed in sh$W T_{\text {apigenin }}$ mice (Figure 1), while simultaneously mitigating histological scores (Figure 2c,d). Collectively, cohousing experiment revealed that the protective effect of apigenin against colitis was communicable, suggesting that systemic administration of apigenin may protect the mucosa from overwhelming inflammation by regulating the composition of the gut microbiota. 


\section{Apigenin treatment affects the composition of the gut microbiota through an intact NIrp6 signaling pathway}

By $16 \mathrm{~S}$ rRNA gene sequencing and subsequent analyses, we next addressed whether the anti-inflammatory role of apigenin was associated with alterations in the gut bacterial composition. Statistical analysis of similarity on Jaccard distances revealed that the gut microbiota composition of sh- $W T_{\text {apigenin }}$ mice was markedly different from the one of controls $\left(R^{2}=0.33\right.$, $P=0.015$; Figure 3a). Employing linear discriminant analysis effect size determination revealed an expansion of several bacterial taxa derived from Bacteroidetes group, including Rikenellaceae, Bacteroidales, and Bacteroides, in
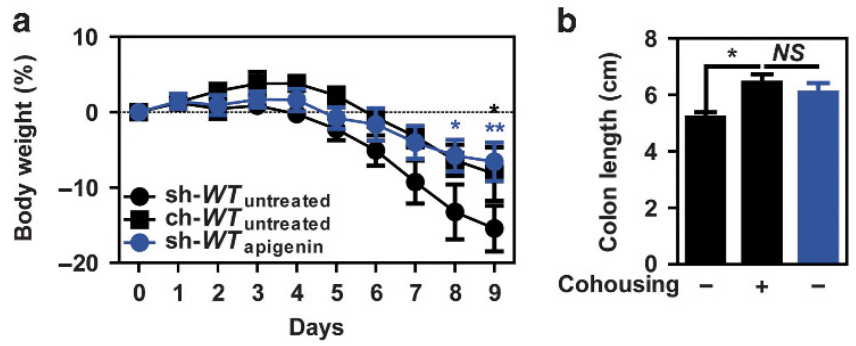

C

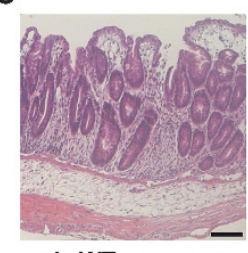

sh-WT untreated

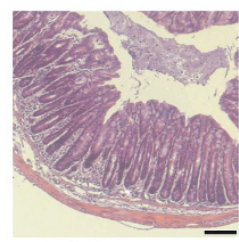

ch-WT untreated d

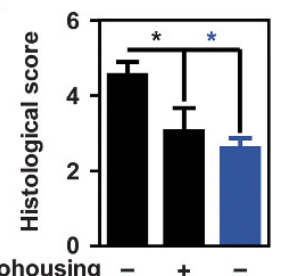

Figure 2 Effective horizontal transmission of the protective effect of apigenin against colitis in mice. Mice were intraperitoneally treated with apigenin at days 20,17, 14, 10, 7, and 3 before induction of colitis by administration of $2 \%$ dextran sulfate sodium (DSS; w/v). Mice were either single-housed ( $N=4$ untreated mice and $N=4$ treated mice) or cohoused ( $N=4$ untreated mice) during experiment as indicated on the figure. (a) Body weight loss, (b) colon lengths, (c) representative hematoxylin and eosin (H\&E)-stained colonic sections, and (d) histological scores are presented. Error bars show s.e.m. ${ }^{\star} P \leq 0.05$ and ${ }^{\star *} P \leq 0.01$. NS, not significant. Scale bar $=00 \mu \mathrm{m}$. Results are representative of two independent experiments. apigenin-treated mice. Furthermore, apigenin treatment in wild-type mice led to a reduction of several bacterial operational taxonomic units derived from Clostridium and Lachnospiraceae (Figure $3 \mathbf{b}$ ). In accordance with previous findings, the bacterial communities among cohoused mice were found similar as determined by principal coordinates analysis and analysis of similarity $(P=0.1515)$. This result suggests that the apigenin-induced changes in the gut microbiota harbor dominance upon cohousing as a likely consequence of coprophagy (Supplementary Figure S1 online). In addition, a trend of higher bacterial diversity and richness was observed in the feces collected from mice treated with apigenin in comparison with untreated animals (Supplementary Figure S2). To obtain further insights into how apigenin affects the composition of the gut microbiota, we performed additional $16 \mathrm{~S}$ rRNA gene sequencing analysis of feces from mice that are deficient for Nlrp6 acting as a regulator of gut bacterial ecology (Supplementary Figure S3a). In line with previous findings, ${ }^{16-}$ ${ }^{18}$ large-scale changes in the gut microbiota composition gave rise to a reduced bacterial diversity in the absence of Nlrp6 $(P=0.001$; Supplementary Figure S3b). In contrast to what was observed in wild-type mice, we failed to notice major global changes in the composition of the gut microbiota from Nlrp6deficient mice upon apigenin treatment (analysis of similarity, $P=0.283$; Figure 3a). Collectively, the impact of apigenin on the composition of the gut microbiota was dependent on an intact Nlrp6 signaling pathway.

\section{The transmissible anti-inflammatory effect of apigenin relies on an intact NIrp6 signaling pathway independently of caspase-1/11 and its adaptor Asc}

Given that the modulation of the composition of the gut microbiota by apigenin requires an intact Nlrp6 signaling, we next evaluated whether Nlrp6 is required for the antiinflammatory effect of apigenin that can be acquired after cohousing. In contrast to what was observed in wild-type mice (Figure 2), apigenin treatment failed to improve wasting disease (Figure 4a) and colon length shortening (Figure $\mathbf{4 b}$ ) in a

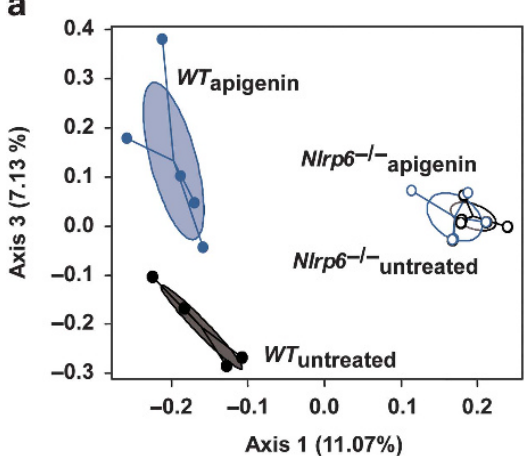

b

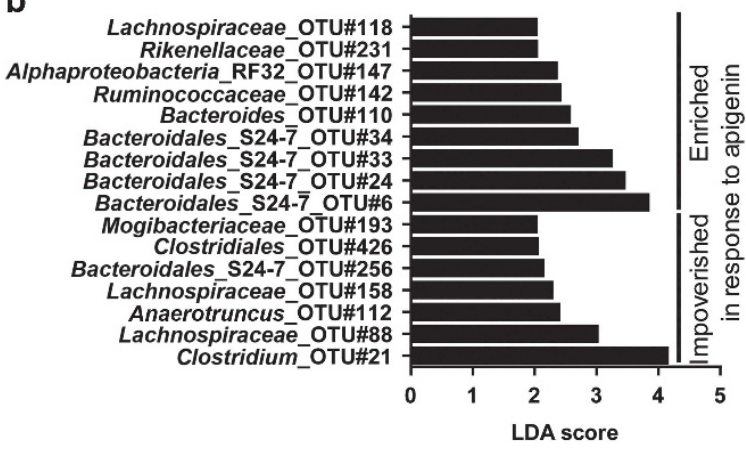

Figure 3 Apigenin shapes the composition of the gut microbiota through NOD-like receptor family pyrin domain containing 6 (Nlrp6) signaling pathway in mice. (a) Principal coordinates analysis of fecal bacterial communities profiled from wild-type and NIrp6 ${ }^{-/-}$mice that were treated or not with apigenin at days $20,17,14,10,7$, and 3 before sampling of feces. Principal coordinates were calculated on nonabundance-based Jaccard distances representing community composition of compared samples. Black and white symbols represent wild-type and NIrp6 $6^{-1-}$ mice, respectively. (b) Analysis of differences in the microbial taxa between apigenin-treated and untreated wild-type animals using LEfSe (linear discriminant analysis (LDA) coupled with effect size measurements). Only bacterial taxa reaching LDA threshold of 2 and $P$ value $\leq 0.05$ are shown. 
mice lacking Nlrp6 (ch-Nlrp6 ${ }^{-/-}$untreated $)$that were cohoused with apigenin-treated Nlrp6-deficient mice (ch-Nlrp $6^{-/-}$apigenin). Consequently, histological scores were not improved in the absence of Nlrp6 as evidenced by both crypt erosion and massive infiltration of inflammatory cells in colonic sections of ch-Nlrp $6^{-/-}$untreated mice with DSS treatment (Figure 4c,d), indicating that the reprogramming of the gut microbiota by apigenin requires a functional Nlrp6 signaling for its dominant transmissibility. Similarly, experiments with single-housed Nlrp6-deficient mice failed to reveal any differences in protection from colitis between apigenin-treated and untreated animals as determined by quantifying body weight loss (Figure 5a), colon length (Figure 5b), and the inflammatory response in subsequent histological analysis (Figure 5c,d). To get further insights into how Nlrp6 signaling may protect mice against acute colitis in response to apigenin, we next determined whether apigenin may protect against DSSinduced colitis through an inhibition of interleukin- $1 \beta$ secretion as a potential consequence of caspase-1/11 inactivation (Figure 1c). Indeed, the inflammasome assembly in macrophages is disrupted in response to apigenin, ${ }^{19}$ suggesting that the protective effect of apigenin would be lost in the absence of the apoptosis-associated specklike protein containing a caspase-recruitment domain (namely Asc encoded by Pycard gene). To this end, Pycard-deficient (Pycard ${ }^{-/-}$) mice were subjected to DSS as previously described. Unexpectedly, $\sim 19 \%$ of initial body weight of Pycard $^{-/-}$mice was lost at day 9 (Figure 5e), whereas apigenin treatment in mutant mice significantly reduced body
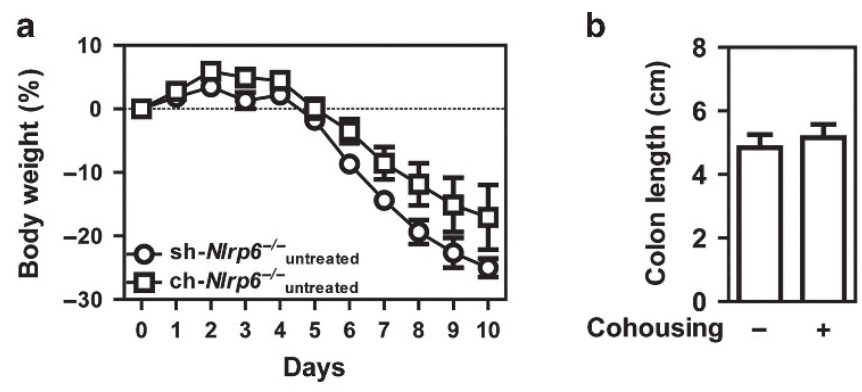

c
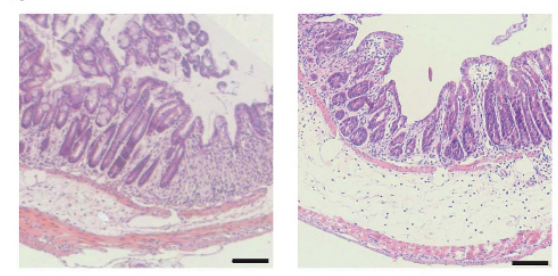

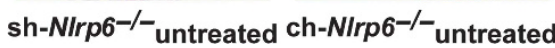

d

Figure 4 NOD-like receptor family pyrin domain containing 6 (NIrp6)deficient mice are resistant to the dominantly transmitted protection conferred by apigenin. NIrp6 $6^{-/-}$mice treated with apigenin at days 20,17 , $14,10,7$, and 3 before induction of colitis by administration of $2 \%$ dextran sulfate sodium (DSS; w/v) when being cohoused with untreated NIrp6 ${ }^{-1-}$ mice. (a) Body weight loss, (b) colon lengths, (c) representative hematoxylin and eosin (H\&E)-stained colonic sections, and (d) histological scores are presented (ch-Nlrp6 ${ }^{-/-}$untreated $N=4$, ch-NIrp6 ${ }^{-1-}$ apigenin $N=5$ ). Error bars show s.e.m. Scale bar $=100 \mu \mathrm{m}$. weight loss (Figure 5e) and colon length shortening (Figure 5f) as what was observed in similarly treated wild-type animals (Figure 1). Consistently, histological investigation showed reduced infiltration of inflammatory cells in apigenin-treated mice that are deficient for Pycard (Figure 5g,h). To verify these results, we next evaluated the efficacy of apigenin in mice that are deficient for both caspase-1 (encoded by the Casp1 gene) and caspase-11 (encoded by the Casp11 gene) (referred herein as Casp1/11 $11^{-/-}$mice). Consistent with the results observed with Pycard $^{-/-}$mice, disease severity was improved in apigenin-treated Casp $1 / 11^{-/-}$mice when compared with untreated mutant animals (Supplementary Figure S4a-d). Consistently, the level of interleukin-18 was not linked at day 10 to the improved disease severity that is found dominantly transmissible upon cohousing with apigenin-treated mice while also having no effect on the secretion of either tumor necrosis factor- $\alpha$ or interleukin-6 (Supplementary Figure S4e-g). Collectively, Nlrp6 signaling determines to a large extent how apigenin protects mice from intestinal inflammation by modulating the composition of the gut microbiota independently of inflammasome activation.

\section{An intact NIrp6 signaling pathway is required for a transmissible antiproliferative effect of apigenin on intestinal epithelial cells}

We next evaluated how the colonic mucosa is conditioned by the specific changes in the gut microbiota that were triggered by apigenin. To this end, we examined the transcript levels of Nlrp6 by quantitative PCR analysis together with those of genes encoding for arginase- 2 and nitric oxide synthase 2 , as markers of M2 and M1 macrophages, respectively. It is indeed worth noting that macrophage polarization by apigenin requires peroxisome proliferator-activated receptor- $\gamma$ activation $^{20}$ that regulates Nlrp6 expression in intestinal epithelial cells. ${ }^{21,22}$ Although whereas a greater expression of Nlrp6 was observed in the colon of sh- $W T_{\text {apigenin }}$ and ch- $W T_{\text {untreated }}$ mice (Figure 6a), the relative ratio of nitric oxide synthase 2 to arginase-2 was significantly enhanced (Figure 6b). Accordingly, the expression of interleukin-10 was significantly reduced upon apigenin administration in wildtype mice (Supplementary Figure S4a). Similarly, the level of interleukin-18 mRNA transcript in the colon of apigenintreated mice was concordantly lowered to the same extent observed in Nlrp6-deficient mice (Supplementary Figure S4b). These results suggested that apigenin may modulate macrophage polarization in the intestine as what was observed in the liver and the adipose tissue of obese mice. ${ }^{20}$ Based on the role of M1 macrophages on epithelial differentiation through the Notch pathway, ${ }^{23}$ we next examined the impact of apigenin on the expression of a canonical target gene of the Notch pathway that encodes for Hairy and Enhancer of Split 1 (Hes1) and on the proliferative index of intestinal epithelial cells by staining the colonic sections for Ki67 before any induction of colitis by DSS administration. In line with previous findings, ${ }^{24}$ a reduced transcriptional expression of Hes1 that inhibits secretory lineage specification coincided with 


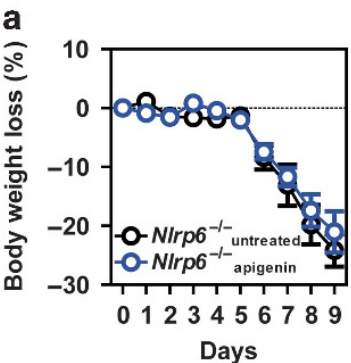

b
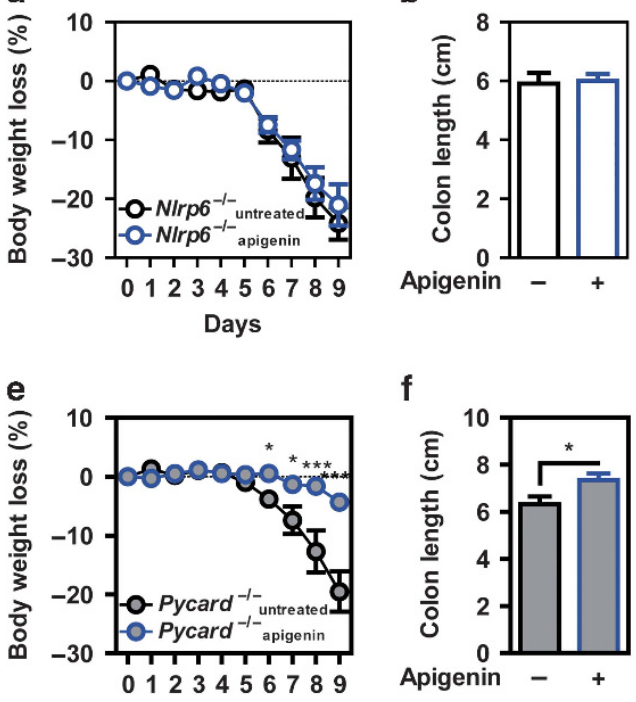

Days

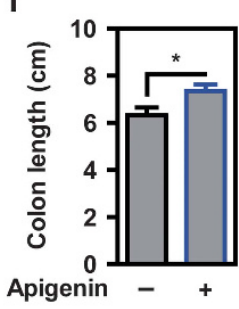

c

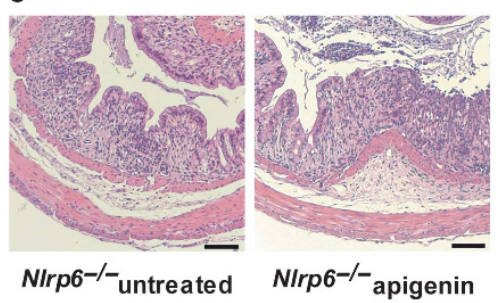

g d

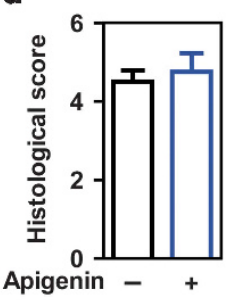

h

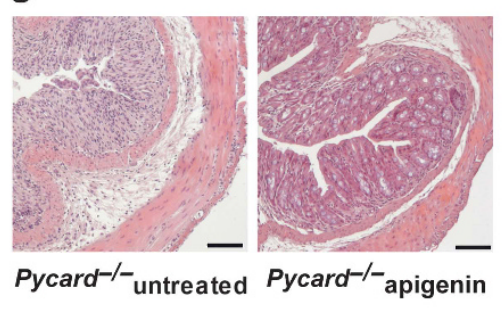

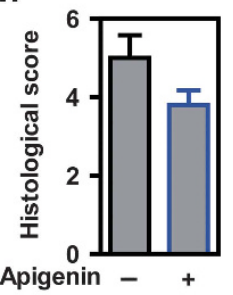

Figure 5 The anti-inflammatory properties of apigenin depends on NOD-like receptor family pyrin domain containing 6 (NIrp6) signaling independently of inflammasome activation in a preclinical model of acute colitis. Single-housed NIrp6 ${ }^{-1-}$ and Pycard ${ }^{-1-}$ mice were treated with apigenin at days 7 and 3 before induction of colitis by $2 \%$ dextran sulfate sodium (DSS; w/v). (a) Body weight loss, (b) colon lengths, (c) representative hematoxylin and eosin (H\&E)-stained colonic sections, and (d) histological scores are presented for Nlrp6 ${ }^{-/-}$mice (N/rp6 ${ }^{-/-}{ }_{\text {untreated }} N=4, N / r p 6^{-/-}$apigenin $\left.N=4\right)$. (e) Body weight loss, (f) colon lengths, (g) representative H\&E-stained colonic sections. and (h) histological scores are depicted for Pycard ${ }^{-1-}$ mice (Pycard $^{-1-}$ untreated $N=4$, Pycard ${ }^{-1-}$ apigenin $\left.N=5\right)$. Error bars show s.e.m. ${ }^{\star} P \leq 0.05$ and ${ }^{* * *} P \leq 0.001$. Scale bar $=100 \mu \mathrm{m}$.
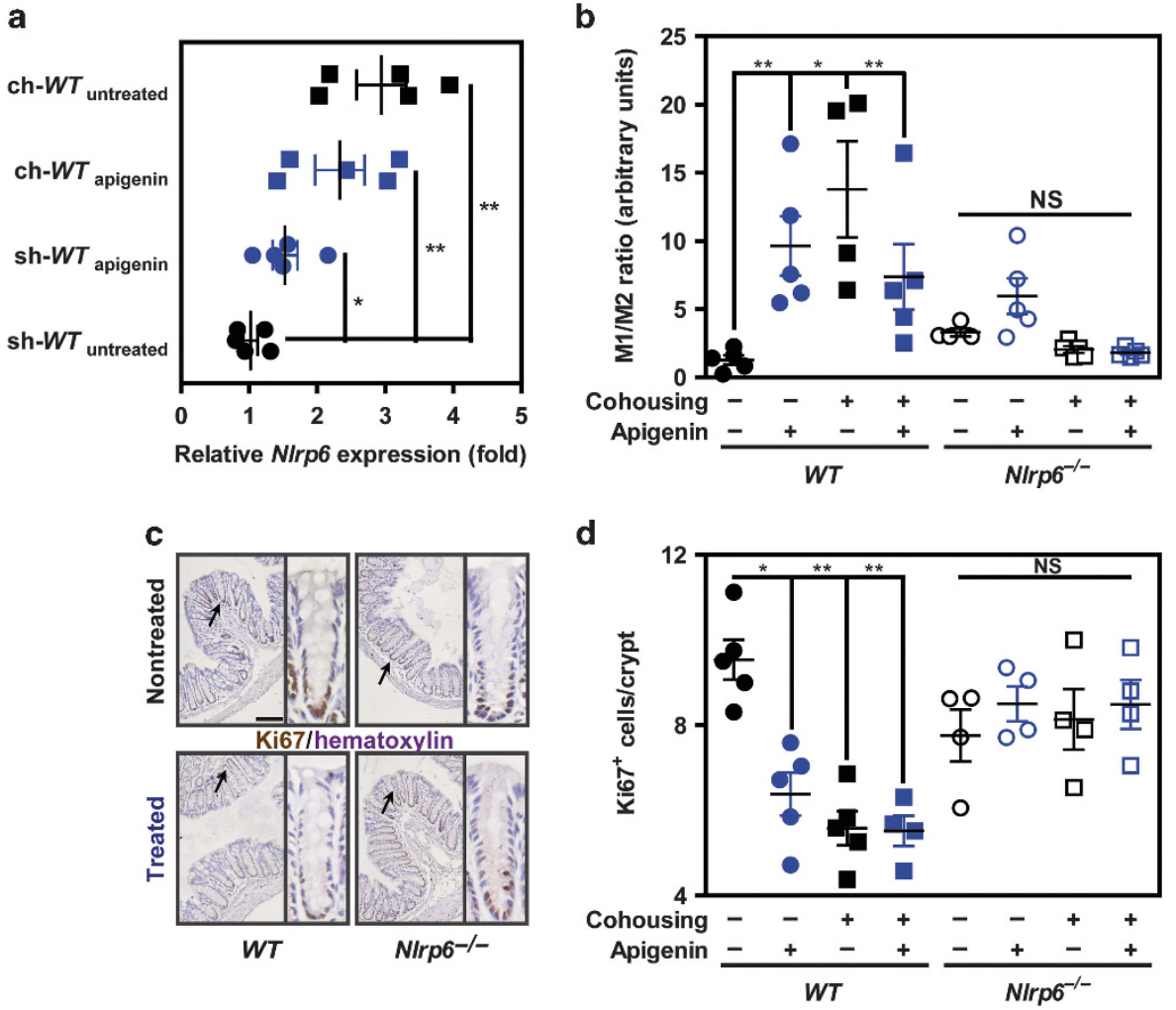

Figure 6 Apigenin promotes NOD-like receptor family pyrin domain containing 6 (NIrp6) expression in the colon and lowers the proliferation rate of intestinal epithelial cells (IECs) in response to compositional changes in the gut microbiota downstream of Nlrp6 signaling before colitis. Single-housed and cohoused wild-type (WT) mice ( $N=5$ for each group) were treated (sh- $W T_{\text {apigenin }}$ and ch- $W T_{\text {apigenin }}$ respectively) or not (sh- $W T_{\text {untreated }}$ and ch$W T_{\text {untreated }}$ respectively) with apigenin over a 3-week period before colitis. (a) Relative expression level of NIrp6 gene measured in the colon. (b) M1/M2 ratio calculated from Nos2 (M1) and Arg2 (M2) expression levels in the colon. (c) Representative Ki67 immunostaining in colonic tissue samples. Scale bar $=100 \mu \mathrm{m}$. (d) Average numbers of Ki67 ${ }^{+}$cells per colonic crypt (nontreated $N=4$ in black, apigenin-treated $N=5$ in blue). Black and white symbols represent WT and NIrp6-deficient mice, respectively. All values are mean \pm s.e.m. ${ }^{\star} P \leq 0.05{ }^{\star \star} P \leq 0.01$. NS, not significant. 
a drop in the number of proliferating epithelial cells in the colon of apigenin-treated wild-type mice when compared with control animals (Supplementary Figure S4c and Figure $\mathbf{6 c}, \mathbf{d}$ ), yet the number of cleaved caspase-3-positive cells was found similar (Supplementary Figure S5). Foremost, we noticed a reduced proliferation index of apigenin-treated mice that was dominantly transmitted after cohousing of control animals (Figure 6d). This finding is in agreement with the diminished expression of Hes1 and the greater abundance of several butyrate-producing bacteria in adult wild-type mice that were treated with apigenin (Figure $3 \mathbf{b}$ ). While being abundantly produced by bacterial fermentation of fiber in the colon, butyrate is an anti-inflammatory short-chain fatty acid that regulates intestinal macrophage function ${ }^{25}$ and has a suppressive effect on proliferation of intestinal epithelial cells. ${ }^{26}$ In contrast, the expression of Hes1 and the antiproliferative effect of apigenin was abrogated in Nlrp6-deficient mice (Supplementary Figure S4c and Figure $\mathbf{6 c}, \mathbf{d}$ ) that were characterized by a lowered abundance of several butyrateproducing bacteria belonging to the Bacteroidetes phylum, ${ }^{27}$ including Alistipes, Barnesiella, and Allobaculum (Supplementary Figure S3c). To explore this Nlrp6-dependent mechanism of apigenin further, we sought to evaluate the role of the stress-induced regenerating islet-derived protein $3 \mathrm{~b}$ (Reg3b). Although being primarily expressed by postmitotic secretory cells in the intestine, ${ }^{28}$ a lowered expression of the c-type lectin Reg3b was noticed in the colon of naive Nlrp6 ${ }^{-1-}$ mice (Supplementary Figure S6). During DSS injury, no protective effect of apigenin on body weight loss (Figure 7a) and colon length shortening (Figure 7b) was observed in Reg3b-deficient mice. Consequently, histological examination of paraffinembedded colon sections (Figure 7c) confirmed the inefficacy of apigenin for protecting Reg3b-deficient mice from extensive villous surface denudation in this acute model of colitis (Figure 7d). Collectively, the protective effect of apigenin against colitis relied on the c-type lectin Reg3b that is regulated by Nlrp6 signaling in the colon.

\section{DISCUSSION}

Apigenin is a secondary metabolite of flavonoids from photosynthetic plants that has been propagated as a natural remedy for protection against several diseases and ailments. Although some bacteria regulate the bioavailability of apigenin, ${ }^{29}$ our results reveal an unexpected mode of action of apigenin on the composition of the gut microbiota downstream of the Nlrp6 signaling pathway. Cohousing experiments revealed a dominant horizontal transmission of the antiinflammatory properties of apigenin that likely depends on the coprophagic and grooming behaviors of mice. Meanwhile, these effects were abrogated in the absence of Nlrp6. Of note, the regulation of the microbiota by Nlrp6 signaling alleviates disease severity in Il10-deficient mice, ${ }^{30}$ whereas the compositional change in the microbiota of Nlrp6-deficient mice results in low taurine and high spermine/histamine levels that contributes to its dominant takeover. ${ }^{31}$ In contrast, the classical inflammasome pathway was not required for the protective

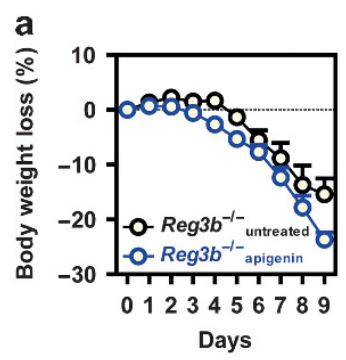

e
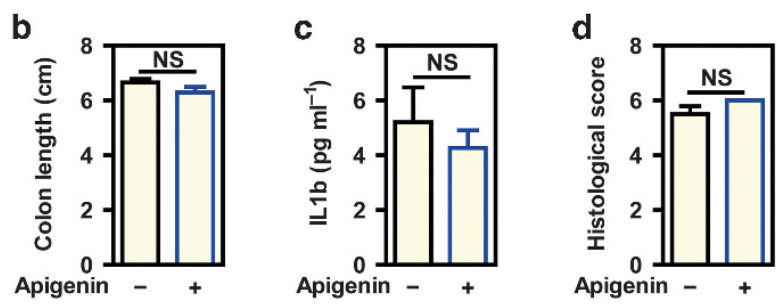

Figure 7 The protective effect of apigenin was compromised in mice that are deficient for the immunomodulatory c-type lectin Reg3b. Single-housed Reg3b-deficient mice were treated at days 7 and $3\left(R e g 3 b^{-1-}\right.$ apigenin $)$ before induction of colitis by $2 \%$ dextran sulfate sodium (DSS; w/v). (a) Body weight loss, (b) colon lengths, (c) interleukin-1 $\beta$ (II1b) levels in colonic explants, (d) histological scores, and (e) representative hematoxylin and eosin (H\&E)-stained colonic sections are presented for mutant mice (Reg3b $b^{-/-}$untreated $N=4, \operatorname{Reg} 3 b^{-/-}$apigenin $N=5$ ). Error bars show s.e.m. ${ }^{\star} P \leq 0.05$. Scale bar $=100 \mu \mathrm{m}$.

effect of apigenin even if secretion of interleukin- $1 \beta$ is found reduced in explants of the intestine from mice that were treated with this flavone (Figure 1c). Equally important, we provide evidence that a lowered proliferation rate of intestinal epithelial cells in the intervillus region is related to an abnormal polarization of macrophages in the gut before colitis. This led to the assumption that the reprogramming effect of apigenin in the gut microbiota results in secretion at physiological concentrations of some antiproliferative bacterial metabolite that skews macrophage polarization, such as butyrate. ${ }^{25,26}$ On this basis, there is now the need to define whether the lack of efficacy of apigenin is caused by a growth disadvantage of some butyrate-producing bacteria in the absence of Nlrp6. Alternatively, it is possible that Nlrp6 signaling may regulate the trafficking and the function of intestinal macrophages that may control the differentiation of secretory cells expressing the c-type lectin Reg3b. In other words, the conditioning of the gut microbiota by apigenin may protect mice against colitis by promoting the accumulation of macrophages that subsequently release factors involved in the maintenance of epithelial integrity, such as interleukin-18 binding protein ${ }^{32}$ and antagonists of the Notch signaling pathway. ${ }^{23}$ Indeed, it has gained broad acceptance that interleukin-18 signaling and Notch activation in intestinal epithelial cells impairs differentiation of secretory lineage (including Paneth cells that preserve intestinal homeostasis ${ }^{33}$ ). In contrast to what we found in the colon of apigenin-treated mice where the proliferation rate of epithelial cells and the expression of some Notch target genes were profoundly decreased, staining of intestinal sections with the proliferation marker Ki67 revealed a marked accumulation of proliferating 
epithelial cells upon constitutive activation of the Notch signaling pathway. ${ }^{24}$ Last but not least, one may also hypothesize that the microbiota-dependent anti-inflammatory effect of apigenin may rely on the regulation of cholesterol metabolism $^{34,35}$ and weight regain after dieting ${ }^{36}$ by Nlrp6 signaling. Collectively, our study demonstrates that the protective action of a common dietary flavone on intestinal inflammation depends on the expression of NOD-like receptor Nlrp6 and of the c-type lectin Reg3b for potentially preventing bacterial colonization of the intestinal mucosa. ${ }^{37}$ The protective effect is independent of the classical inflammasome function mediated via caspase-1/11 and Asc, but involves preservation of epithelial barrier integrity as a response to compositional changes of the gut microbiota downstream of Nlrp6 signaling.

\section{METHODS}

Mice. All animal studies were approved by the local investigational review board (CEEA232009R) in an accredited establishment (No. B59-108) according to governmental guidelines No. 86/609/CEE. Ageand gender-matched Nlrp6-deficient $\left(\mathrm{Nlrp6}^{-/-}\right)$, Caspase 1/11deficient (Casp1/11 ${ }^{-/-}$mice), Asc-deficient $\left(\right.$Pycard $\left.^{-/-}\right)$, Reg3bdeficient $\left(\operatorname{Reg} 3 b^{-1-}\right)$, and control C57BL6/J mice had free access to a standard laboratory chow diet in a half-day light cycle exposure and temperature-controlled environment. Colitis was induced by giving mice $2 \%(\mathrm{w} / \mathrm{v})$ DSS (TdB Consultancy, Uppsala, Sweden) for a period of 7 days followed by drinking water for 2 to 3 days. DSS was dissolved in autoclaved drinking water. Signs of morbidity, including body weight, stool consistency and occult blood, or the presence of macroscopic rectal bleeding gross, were monitored daily as previously described. ${ }^{38}$ Apigenin $(25 \mu \mathrm{g}$ per mouse; Santa Cruz, Heidelberg, Germany) was given intraperitoneally twice a week for 1-3 weeks before the induction of colitis. For cohousing experiments, mice were kept in the same cage for a period of 3 weeks before exposure to DSS and left together during colitis. At the end of the challenge, mice were autopsied to assess the severity of the disease by measurement of colon lengths and by histological scoring. At $1 \mathrm{~cm}$ above the anal canal, a resection specimen of the distal colon was dissected out and kept frozen in RNAlater (Courtaboeuf, France) until subsequent RNA isolation for quantitative PCR analyses. Tissue specimens were also collected and kept frozen until further quantification of protein levels and western blotting analysis. Finally, a remaining piece of distal colon was fixed in $4 \%$ paraformaldehyde and embedded in paraffin for immunohistochemistry analysis by two investigators.

Histological scoring and immunohistochemistry. Histological scoring of hematoxylin and eosin-stained sections take into account the level of inflammatory cell infiltration and the epithelial damage as previously described. ${ }^{38}$ Intestinal tissue was fixed in formalin and dehydrated in series of increasing concentration of alcohols and toluene before being embedded in paraffin. For immunohistochemistry, $5 \mu \mathrm{m}$-thick tissue sections were placed on Superfrost Plus slides (Thermo Scientific, Waltham, MA), incubated for $10 \mathrm{~min}$ at $60{ }^{\circ} \mathrm{C}$, and rehydrated through a series of graded alcohols and distilled water. Endogenous peroxidases were blocked by $3 \%$ hydrogen peroxide for $10 \mathrm{~min}$. Antigen retrieval was performed in citrate buffer ( $10 \mathrm{~mm}, \mathrm{pH} 6)$ by steaming sections in a microwave oven for $20 \mathrm{~min}$. Tissue sections were blocked with $5 \%$ bovine serum albumin/ phosphate-buffered saline for $30 \mathrm{~min}$ at room temperature and primary antibody against either Ki67 (1:100, ab15580, Abcam, Paris, France) or cleaved caspase-3 (1:50, 9661, Cell Signaling Technology, Leiden, The Netherlands) was directly applied and incubated for $1 \mathrm{~h}$ at room temperature or overnight at $4{ }^{\circ} \mathrm{C}$, respectively. Slides were washed three times in phosphate-buffered saline before applying secondary peroxidase-conjugated goat anti-rabbit IgG (1:100,
Interchim, Montluçon, France) antibody for $2 \mathrm{~h}$ at room temperature. Targeted antigens were visualized by using $3.3^{\prime}$-diaminobenzidine solution (BD Pharmingen, Le Pont de Claix, France) followed by nuclear counterstain with hematoxylin. Cells that are positive for either Ki67 or cleaved caspase- 3 were counted for all intact crypts from each section.

Enzyme-linked immunosorbent assay. Protein levels of interleukin$1 b$, interleukin-18, interleukin- 6 , and tumor necrosis factor- $\alpha$ were determined from the supernatants of colon explants by specific enzyme-linked immunosorbent assay according to the manufacturer's instructions (R\&D System, Lille, France).

Microbiota analysis. The $16 \mathrm{~S}$ rRNA gene variable region V3 and V4 was amplified using dual indexed fusion primers; in brief, primers consist of Illumina (San Diego, CA) linker sequence, 12 base barcode sequence, and heterogeneity spacer followed by either $16 \mathrm{~S}$ rRNA genespecific forward (319F: $5^{\prime}$-ACTCCTACGGGAGGCAGCAG-3') or reverse (806R: 5'-GGACTACHVGGGTWTCTAAT-3') primer sequences. ${ }^{39}$ DNA was amplified using above-mentioned composite primers in duplicate in a GeneAmp PCR system 9700 (Applied Biosystems, Foster City, CA) using the following cycling conditions: an initial denaturation of $3 \mathrm{~min}$ at $98^{\circ} \mathrm{C}$ followed by 30 cycles, denaturation at $98^{\circ} \mathrm{C}$ for $10 \mathrm{~s}$, annealing at $50^{\circ} \mathrm{C}$ for $30 \mathrm{~s}$, and elongation at $72{ }^{\circ} \mathrm{C}$ for $30 \mathrm{~s}$. Final extension was at $72^{\circ} \mathrm{C}$ for $10 \mathrm{~min}$. The amplicon size and amplification performance were validated by running the amplified product on agarose gel. Amplicon quantities were normalized using SequalPrep kit (Invitrogen, Darmstadt, Germany) and pooled as a single library. Sequencing was performed using Illumina MiSeq using $2 \times 300$ sequencing kit with standard HP10 and HP11 primers. Sequencing reads were primarily processed for quality control using the software MOTHUR (https://www.mothur.org). Forward and reverse reads were assembled to form contigs, and reads not having perfectly matched specific primers and barcodes, having any ambiguous base, and more than 8 homopolymers were removed for subsequent analysis. Sequences not aligning to desired bacterial V3V4 variable regions, suspected chimera, and likely to be of nonbacterial origin were detected and removed. Sequences with at least 97\% similarity were clustered into operational taxonomical units. These operational taxonomical units were classified taxonomically using Mothur modified Greengenes reference and taxonomy database. ${ }^{40}$ Principle coordinate analysis was performed on Jaccard distances among communities. Principle coordinates were visualized using rgl with in $\mathrm{R}$ application package (www.r-project.org). LEfSe (linear discriminant analysis with effect size) was implemented to identify operational taxonomical units differentially enriched or deleted in apigenin-treated and untreated animals. ${ }^{41}$ LEfSe analysis was performed in Mothur using 1000 bootstrap iteration for linear discriminant analysis score calculation. Observed number of phylotypes, Shannon index, nonparametric estimate of Shannon diversity index, and Chaol richness and phylodiversity were calculated within Mothur as parameters of $\alpha$-diversity indices in apigenin-treated and untreated wild-type mice.

Gene expression. Isolated RNA was reverse-transcribed with the complementary DNA synthesis kit (Agilent Technologies, Les Ulis, France) according to the manufacturer's instructions. The resulting complementary DNA (equivalent to $500 \mathrm{ng}$ of total RNA) was amplified using the SYBR Green real-time PCR kit and detected on a Stratagene Mx3005P (Agilent Technologies). Quantitative PCR was performed using forward and reverse primers (sequences available upon request). On completion of the PCR amplification, a DNA melting curve analysis was carried out in order to confirm the presence of a single amplicon. Actb was used as an internal reference gene in order to normalize the transcript levels. Relative mRNA levels (2DDCt) were determined by comparing (i) the PCR cycle thresholds (Ct) for the gene of interest and Actb (DCt) and (ii) DCt values for treated and control groups (DDCt). 
Statistics. Data were analyzed using Prism4.0 (GraphPad Software, San Diego, CA). The nonparametric Kruskal-Wallis test with Dunn's multiple comparison test, the nonparametric Mann-Whitney test, or the nonparametric two-way analysis of variance test with Bonferroni's multiple comparison test were used. Values represent the mean of normalized data \pm s.e.m. Asterisk indicates significant difference $P \leq 0.05$.

SUPPLEMENTARY MATERIAL is linked to the online version of the paper at http://www.nature.com/mi

\section{ACKNOWLEDGMENTS}

This work was supported by grants from the Fondation pour la Recherche Médicale (DEQ20130326475) for M.C. and from CNRS and European Regional Development Fund (FEDER n 2016-00110366) for B.R. S.N. was a recipient of a postdoctoral fellowship from the Ligue contre le Cancer. N.W. was a recipient of a postdoctoral fellowship from Agence Nationale de la Recherche (ANR-13-BSV3-0014-01). P.R. was supported by the Cluster of Excellence Inflammation at Interfaces, RTG1743 (TP5), and the SFB877 (B9). We thank K. Jambou, C. Bertrand, T. Grandjean, C. Bondu, M. Nesterenko, and B. Duchêne for excellent technical assistance.

\section{AUTHOR CONTRIBUTIONS}

K.R. and S.N. performed the majority of the experimental procedures. A.R. and P.R. contributed to the microbiota analysis. A.D.-C. contributed to cell culture experiments and hematoxylin and eosin staining. M.D. and J.C. performed quantitative reverse transcription-PCR and enzyme-linked immunosorbent assay analysis, respectively. N.W. and K.R. contributed to Ki67 staining of colon sections. All authors contributed to interpretation of raw data and critically reviewed and/or modified the manuscript. M.C. conceived, designed, and wrote the paper.

\section{DISCLOSURE}

The authors declared no conflict of interest.

c 2018 Society for Mucosal Immunology

\section{REFERENCES}

1. Ley, R.E. et al. Evolution of mammals and their gut microbes. Science $\mathbf{3 2 0}$, 1647-1651 (2008).

2. Scanlan, P.D., Shanahan, F., O'Mahony, C. \& Marchesi, J.R. Cultureindependent analyses of temporal variation of the dominant fecal microbiota and targeted bacterial subgroups in Crohn's disease. J. Clin. Microbiol. 44, 3980-3988 (2006).

3. Manichanh, C. et al. Reduced diversity of faecal microbiota in Crohn's disease revealed by a metagenomic approach. Gut 55, 205211 (2006).

4. Lamas, B. et al. CARD9 impacts colitis by altering gut microbiota metabolism of tryptophan into aryl hydrocarbon receptor ligands. Nat. Med. 22, 598-605 (2016).

5. Gonzalez, R. et al. Effects of flavonoids and other polyphenols on inflammation. Crit. Rev. Food Sci. Nutr. 51, 331-362 (2011).

6. Lopez-Posadas, R. et al. Flavonoids exert distinct modulatory actions on cyclooxygenase 2 and NF-kappaB in an intestinal epithelial cell line (IEC18). Br. J. Pharmacol. 160, 1714-1726 (2010).

7. Seo, H.S. et al. Apigenin induces caspase-dependent apoptosis by inhibiting signal transducer and activator of transcription 3 signaling in HER2-overexpressing SKBR3 breast cancer cells. Mol. Med. Rep. 12, 2977-2984 (2015).

8. Xu, X., Li, M., Chen, W., Yu, H., Yang, Y. \& Hang, L. Apigenin attenuates oxidative injury in ARPE-19 cells thorough activation of Nif2 pathway. Oxid. Med. Cell Longev. 2016, 4378461 (2016).

9. Hoensch, H., Richling, E., Kruis, W. \& Kirch, W. [Colorectal cancer prevention by flavonoids]. Med. Klin. 105, 554-559 (2010).

10. Banerjee, K. \& Mandal, M. Oxidative stress triggered by naturally occurring flavone apigenin results in senescence and chemotherapeutic effect in human colorectal cancer cells. Redox Biol. 5, 153-162 (2015).
11. Mascaraque, C., Gonzalez, R., Suarez, M.D., Zarzuelo, A., Sanchez de Medina, F. \& Martinez-Augustin, O. Intestinal anti-inflammatory activity of apigenin $\mathrm{K}$ in two rat colitis models induced by trinitrobenzenesulfonic acid and dextran sulphate sodium. Br. J. Nutr. 113, 618-626 (2015).

12. Marquez-Flores, Y.K., Villegas, I., Cardeno, A., Rosillo, M.A. \& Alarcon-dela-Lastra, C. Apigenin supplementation protects the development of dextran sulfate sodium-induced murine experimental colitis by inhibiting canonical and non-canonical inflammasome signaling pathways. J. Nutr. Biochem. 30, 143-152 (2016).

13. Ganjare, A.B., Nirmal, S.A. \& Patil, A.N. Use of apigenin from Cordia dichotoma in the treatment of colitis. Fitoterapia 82, 1052-1056 (2011).

14. Vezza, T., Rodriguez-Nogales, A., Algieri, F., Utrilla, M.P., RodriguezCabezas, M.E. \& Galvez, J. Flavonoids in inflammatory bowel disease: a review. Nutrients 8, 211 (2016).

15. Shin, E.K., Kwon, H.S., Kim, Y.H., Shin, H.K. \& Kim, J.K. Chrysin, a natural flavone, improves murine inflammatory bowel diseases. Biochem. Biophys. Res. Commun. 381, 502-507 (2009).

16. Elinav, E. et al. NLRP6 inflammasome regulates colonic microbial ecology and risk for colitis. Cell 145, 745-757 (2011).

17. Normand, S. et al. Nod-like receptor pyrin domain-containing protein 6 (NLRP6) controls epithelial self-renewal and colorectal carcinogenesis upon injury. Proc. Natl. Acad. Sci. USA 108, 9601-9606 (2011).

18. Chen, G.Y., Liu, M., Wang, F., Bertin, J. \& Nunez, G. A functional role for NIrp6 in intestinal inflammation and tumorigenesis. J. Immunol. 186, 71877194 (2011).

19. Zhang, X., Wang, G., Gurley, E.C. \& Zhou, H. Flavonoid apigenin inhibits lipopolysaccharide-induced inflammatory response through multiple mechanisms in macrophages. PLOS ONE 9, e107072 (2014).

20. Feng, X. et al. Activation of PPARgamma by a natural flavonoid modulator, apigenin ameliorates obesity-related inflammation via regulation of macrophage polarization. EBioMedicine 9, 61-76 (2016).

21. Sun, Y. et al. Stress-induced corticotropin-releasing hormone-mediated NLRP6 inflammasome inhibition and transmissible enteritis in mice. Gastroenterology 144, 1478-1487 e1478 (2013).

22. Kempster, S.L. et al. Developmental control of the Nirp6 inflammasome and a substrate, IL-18, in mammalian intestine. Am. J. Physiol. Gastrointest. Liver Physiol. 300, G253-G263 (2011).

23. Ortiz-Masia, D. et al. M1 macrophages activate notch signalling in epithelial cells: relevance in Crohn's disease. J. Crohns Colitis 10, 582-592 (2016).

24. Fre, S., Huyghe, M., Mourikis, P., Robine, S., Louvard, D. \& ArtavanisTsakonas, S. Notch signals control the fate of immature progenitor cells in the intestine. Nature 435, 964-968 (2005).

25. Chang, P.V., Hao, L., Offermanns, S. \& Medzhitov, R. The microbial metabolite butyrate regulates intestinal macrophage function via histone deacetylase inhibition. Proc. Natl. Acad. Sci. USA 111, 22472252 (2014)

26. Kaiko, G.E. et al. The colonic crypt protects stem cells from microbiotaderived metabolites. Cell 165, 1708-1720 (2016).

27. Vital, M., Howe, A.C. \& Tiedje, J.M. Revealing the bacterial butyrate synthesis pathways by analyzing (meta)genomic data. mBio 5, e00889 (2014).

28. Gironella, M. et al. Anti-inflammatory effects of pancreatitis associated protein in inflammatory bowel disease. Gut 54, 1244-1253 (2005).

29. Simons, A.L., Renouf, M., Hendrich, S. \& Murphy, P.A. Human gut microbial degradation of flavonoids: structure-function relationships. J. Agric. Food Chem. 53, 4258-4263 (2005).

30. Seregin, S.S. et al. NLRP6 protects $1110-/$ - mice from colitis by limiting colonization of Akkermansia muciniphila. Cell Rep. 19, 733-745 (2017).

31. Levy, M. et al. Microbiota-modulated metabolites shape the intestinal microenvironment by regulating NLRP6 inflammasome signaling. Cell 163, 1428-1443 (2015).

32. Wlodarska, M. et al. NLRP6 inflammasome orchestrates the colonic hostmicrobial interface by regulating goblet cell mucus secretion. Cell 156, 1045-1059 (2014).

33. Adolph, T.E. et al. Paneth cells as a site of origin for intestinal inflammation. Nature 503, 272-276 (2013).

34. Ren, B. et al. Apigenin and naringenin regulate glucose and lipid metabolism, and ameliorate vascular dysfunction in type 2 diabetic rats. Eur. J. Pharmacol. 773, 13-23 (2016). 
35. Jung, U.J., Cho, Y.Y. \& Choi, M.S. Apigenin ameliorates dyslipidemia, hepatic steatosis and insulin resistance by modulating metabolic and transcriptional profiles in the liver of high-fat diet-induced obese mice. Nutrients 8, pii: E305 (2016).

36. Thaiss, C.A. et al. Persistent microbiome alterations modulate the rate of post-dieting weight regain. Nature; doi: 10.1038/nature20796; e-pub ahead of print 24 November 2016 (2016).

37. Wang, L. et al. Intestinal REG3 lectins protect against alcoholic steatohepatitis by reducing mucosa-associated microbiota and preventing bacterial translocation. Cell Host Microbe 19, 227-239 (2016).
38. Wirtz, S., Neufert, C., Weigmann, B. \& Neurath, M.F. Chemically induced mouse models of intestinal inflammation. Nat. Protoc. 2, 541-546 (2007).

39. Fadrosh, D.W. et al. An improved dual-indexing approach for multiplexed $16 \mathrm{~S}$ rRNA gene sequencing on the lllumina MiSeq platform. Microbiome 2 , 6 (2014).

40. DeSantis, T.Z. et al. Greengenes, a chimera-checked 16S rRNA gene database and workbench compatible with ARB. Appl. Environ. Microbiol. 72, 5069-5072 (2006).

41. Segata, N. et al. Metagenomic biomarker discovery and explanation. Genome Biol. 12, R60 (2011). 